\title{
Radiografía de las agencias que ofrecen servicios externos de comunicación estratégica y relaciones públicas en regiones periféricas: el caso gallego
}

\section{María Isabel Míguez-González ${ }^{1}$ Carmen Costa-Sánchez ${ }^{2}$ Miguel Túñez-López ${ }^{3}$}

Recibido: 2017-09-06

Enviado a pares: 2017-09-14
Aprobado por pares: 2017-09-20

Aceptado: 2018-01-21

DOI: 10.5294/pacla.2019.22.1.5

Para citar este artículo / to reference this article / para citar este artigo Míguez-González, M. I., Costa-Sánchez, C. y Túñez-López, M. (2019). Radiografía de las agencias que ofrecen servicios externos de comunicación estratégica y relaciones públicas en regiones periféricas: el caso gallego. Palabra Clave 22(1), e2215. D0I: 10.5294/ pacla.2019.22.1.5

\section{Resumen}

El presente estudio tiene por objetivo describir las características y peculiaridades de las agencias que ofrecen servicios de comunicación estratégica en una comunidad periférica como Galicia, profundizar en la evolución de la oferta y demanda de estos servicios, y valorar el contexto económico como condicionante de la actividad de las agencias. En cuanto a la metodología, la investigación se apoya en un cuestionario en línea distribuido a través del correo electrónico a los responsables de agencias gallegas que ofertan servicios de comunicación. Los resultados muestran un sector en el que las empresas, por lo general, pequeñas agencias de publicidad o servicios plenos dirigidas por varones con formación universitaria, aunque su

\footnotetext{
orcid.org/0000-0002-0580-8493. Universidade de Vigo, España. mabelm@uvigo.es

2 orcid.org/0000-0001-8154-9537. Universidade da Coruña, España. carmen.costa@udc.es

3 orcid.org/0000-0002-5036-9143. Universidade de Santiago de Compostela, España. miguel.tunez@usc.es
} 
plantilla sea mayoritariamente femenina, se crean y destruyen con facilidad, condicionadas aun por las secuelas de la crisis económica y por el reducido tamaño de sus clientes. Si bien la elevada atomización y la falta de especialización son factores diferenciales con respecto a lo que sucede en contextos más amplios, las tendencias en la oferta y demanda de servicios específicos de comunicación son similares a las destacadas por diferentes estudios del ámbito nacional e internacional, con una prevalencia clara de servicios relacionados con internet y una demanda creciente de servicios de tipo estratégico. El estudio apunta, por tanto, a un elevado nivel de globalización en las características de la actividad comunicativa, a pesar de las diferencias estructurales del sector en los contextos periféricos.

\section{Palabras clave (fuente: tesauro de la Unesco)}

Agencias; agencias de comunicación; comunicación estratégica; relaciones públicas; servicios; regiones periféricas; Galicia. 


\section{In-depth Analysis of Agencies Offering Strategic Communication and Public Relations Services in Peripheral Regions: The Galician Case}

\section{Abstract}

This study intends to describe the characteristics and peculiarities of agencies offering strategic communication services in a peripheral community such as Galicia, to delve into the evolution of supply and demand of these services, and to assess the economic context as a determining factor of the business of agencies. As for the method, the research is supported by an online questionnaire distributed via e-mail to the heads of Galician agencies offering communication services. Results show a sector in which companies, generally small advertising or full-service agencies run by men with university education - although their staff is mostly female-, are easily created and liquidated, still conditioned by the aftermath of the economic crisis and the small size of their customers. While high fragmentation and lack of specialization are differential factors with respect to what occurs in broader contexts, the trends in the supply and demand of specific communication services are similar to those noted by national and international studies, with a clear prevalence of internet-related services and a growing demand for strategic services. Therefore, the study points to a high level of globalization in the characteristics of the communicative business, despite the structural differences of the sector in peripheral contexts.

\section{Keywords (source: Unesco Thesaurus)}

Agencies; communication agencies; strategic communication; public relations; services; peripheral regions; Galicia. 


\section{Radiografia das agências que oferecem serviços externos de comunicação estratégica e relações públicas em regiões periféricas: 0 caso galego}

\section{Resumo}

Este estudo tem como objetivo descrever as características e peculiaridades das agências de prestação de serviços de comunicação estratégica em uma comunidade periférica como Galícia, aprofundar na evolução da oferta e da demanda desses serviços, e avaliar a situação económica como condicionante da atividade das agências. Quanto à metodologia, a pesquisa é apoiada por um questionário on-line distribuído via e-mail aos responsáveis das agências galegas que oferecem serviços de comunicação. Os resultados mostram um setor em que as empresas, em geral, pequenas agências de publicidade ou serviços plenos, dirigidas por homens com formação universitária, embora sua força de trabalho seja predominantemente feminina, são criadas e destruídas facilmente, condicionadas pelas sequelas da crise econômica e pelo pequeno tamanho de seus clientes. Enquanto a alta fragmentação e a falta de especialização são fatores diferenciais em relação ao que acontece em contextos mais amplos, as tendências na oferta e procura de serviços de comunicação específicos são semelhantes às destacadas por diversos estudos nacionais e internacionais, com uma prevalência clara de serviços relacionados à internet e uma crescente demanda por serviços estratégicos. $\mathrm{O}$ estudo aponta, portanto, para um alto nível de globalização nas características da atividade comunicativa, apesar das diferenças estruturais do setor nos contextos periféricos.

\section{Palavras-chave (fonte: tesauro da Unesco)}

Agências; agências de comunicação; comunicação estratégica; relações públicas; serviços; região periférica; Galiza. 


\section{Introducción}

El presente estudio tiene por objeto realizar una radiografía cualitativa, aunque a partir de datos cuantificados, de las agencias que ofrecen servicios de comunicación estratégica y relaciones públicas en la comunidad autónoma de Galicia. El trabajo se enmarca en un proyecto mayor promovido por la delegación gallega de la Asociación de Directivos de Comunicación (Dircom) y desarrollado conjuntamente por las tres universidades gallegas (Universidade da Coruña, Universidade de Santiago de Compostela y Universidade de Vigo), para analizar el estado de la actividad de comunicación y relaciones públicas en la comunidad a partir de las respuestas a diferentes cuestionarios por parte de responsables de comunicación de empresas, instituciones y agencias.

Dircom cuenta con una trayectoria dilatada en la elaboración de estudios sobre el estado de la comunicación en España, con un informe quinquenal que ha alcanzado ya su tercera edición (Dircom, 2015) y que interroga a los responsables de comunicación de un elevado número de grandes empresas españolas. Sin embargo, el presente proyecto refleja el interés de la asociación por profundizar en las características de la actividad comunicativa en un ámbito geográficamente más restringido y periférico como es el gallego y en un contexto empresarial e institucional muy diferente, con un tejido de organizaciones de tamaño mucho más reducido, en el que la situación de los agentes que ofrecen servicios de comunicación y relaciones públicas puede diferir considerablemente de la que se presenta para las grandes consultoras de nivel nacional e internacional.

Este estudio no es, no obstante, el primero que trata de entender las dinámicas del sector de la comunicación en Galicia. El Consello da Cultura Galega promueve desde hace más de veinte años, generalmente con una periodicidad bianual, un informe sobre la comunicación en la comunidad. También algunas consultoras han realizado sus aportaciones en este sentido, con informes más parciales como el realizado por MundiNova Consultores de Comunicación (2013) sobre la comunicación empresarial en Galicia. A estos trabajos cabría sumarles otros estudios del 
ámbito académico como el trabajo de García-González y Piñeiro-Otero (2011) sobre el papel de la mujer en las agencias de publicidad gallegas.

Frente a la especificidad de estas investigaciones centradas en el panorama gallego, cabe destacar por su relevancia el European Communication Monitor, principal estudio anual sobre comunicación a nivel europeo que cuenta ya con una edición en 2017 y que sirve como referencia fundamental para la identificación de tendencias y la consideración de variables de análisis en cualquier radiografía sobre la comunicación que se desee realizar.

Tomando como referencia estos y otros estudios previos (Lalueza, 2010; Estanyol y Lalueza, 2014), se han incluido en la investigación diferentes elementos que resultan de interés para conocer el funcionamiento de la actividad comunicativa en Galicia, aportando en algunos casos información novedosa y permitiendo en otros realizar comparaciones con trabajos anteriores, para poder valorar la evolución del sector y la consistencia de los resultados.

Por lo que se refiere a este análisis de las agencias que ofrecen servicios de comunicación y relaciones públicas y que operan en Galicia, cabe destacar que, según el estudio realizado por MundiNova Consultores de Comunicación (2013), "solo el 52 \% de las grandes empresas gallegas cuentan con un director de comunicación (dircom) integrado dentro de su organigrama" y solo el $41 \%$ disponen de departamento de comunicación, lo cual obliga a un elevado porcentaje de empresas a contratar los servicios de agencias externas si desean poder ejecutar alguna actividad comunicativa de un modo profesional; el estudio de MundiNova corrobora que un $58 \%$ de las empresas consultadas recurren de forma habitual u ocasional a los servicios de agencias externas, incluso disponiendo de dircom o departamento de comunicación. Las agencias son, por tanto, un elemento clave en el desarrollo de la actividad comunicativa de empresas e instituciones en Galicia.

Autores como Xifra (2014) o Barquero y Castillo (2011) coinciden en afirmar que en España es en especial frecuente esta tendencia a contratar servicios de comunicación externos, ya por la ausencia de un departamento 
de comunicación interno, situación usual en pequeñas y medianas empresas, ya por la necesidad de complementar la labor de estos departamentos, incluso en el caso de grandes compañías. La contratación de una consultora externa aporta, según Rojas (2008), ventajas como la reducción de costes tanto de producción como de mantenimiento, dada la facilidad para finalizar la relación cuando los servicios no son necesarios o la especialización.

Podría afirmarse que las principales empresas ofertantes de servicios de comunicación son las agencias o consultoras de comunicación o de relaciones públicas. Tal y como apuntan Rodríguez-Salcedo y Buil-Gazol (2015, p. 252), se recurre a diversos términos para definir la naturaleza de este tipo de empresas, las cuales Rojas (2008, p. 68) define como "una empresa que no solo ofrece consultoría estratégica sobre comunicación, tanto a nivel interno como externo, sino que también puede y suele ser la encargada de llevar a cabo el programa que ofrece a sus clientes por unos honorarios determinados". Entre los servicios ofrecidos por este tipo de agencias, Wilcox, Cameron y Xifra (2012, p. 109) mencionan la comunicación de marketing, entendida como la utilización de herramientas de las relaciones públicas para apoyar la venta de productos o servicios, la formación de portavoces, la investigación y evaluación, la comunicación de crisis, el análisis de los medios de comunicación, las relaciones con la comunidad, la organización de eventos, las relaciones institucionales, la gestión de cuestiones de marca y reputación corporativa y las relaciones financieras, así como otros servicios especializados de comunicación que se han desarrollado a medida que han surgido nuevas tendencias, entre los que cabría destacar la comunicación en medios sociales y digitales. Estas agencias han mostrado una evolución muy positiva en los últimos años en España. La facturación media de las treinta agencias más relevantes del país fue de EUR 5,3 millones en 2015, un 10,95\% más que en el ejercicio anterior (PRNoticias y Torres y Carrera consultores de comunicación, 2017).

Sin embargo, limitar el análisis a las agencias de comunicación o relaciones públicas no reflejaría la realidad del sector, ya que este tipo de servicios también son ofertados por agencias de servicios plenos y por un elevado número de agencias de publicidad. 
El primer objetivo del estudio, por tanto, ha sido realizar una descripción básica de las características, no solo de las agencias de comunicación, sino de todas las agencias que ofrecen servicios de comunicación estratégica en Galicia, en función de su tipología, tamaño, antigüedad y perfil personal $y$ formativo de sus trabajadores y responsables. Por lo que se refiere a estos últimos aspectos, se ha tratado de determinar si en las agencias gallegas se refleja la feminización del sector a la que apuntan diversos estudios internacionales (Aldoory y Toth, 2002, 2004; Sha y Toth, 2005; Daugherty, 2014) y si se repiten los patrones formativos habituales detectados en los directores de comunicación de organizaciones a nivel nacional.

El segundo objetivo ha sido profundizar en el tipo de servicios ofertados por las agencias, los demandados por los clientes y las estimaciones previstas de demanda a cinco años vista. En relación con este aspecto, atendiendo a la última edición del monitor europeo de comunicación (Zerfass, Moreno, Tench, Verčič y Verhoeven, 2017), cabe mencionar dos preocupaciones estratégicas prioritarias en estos momentos en la dirección de comunicación en Europa: afrontar la evolución digital y de la web social, y vincular la estrategia empresarial con la comunicación. Estos dos temas fundamentales se combinan con otros retos identificados en el estudio y su relevancia varía considerablemente entre los distintos países en función del grado de desarrollo de sus sistemas de comunicación y de sus circunstancias socioeconómicas. Así, en España, si bien la vinculación de la estrategia empresarial con la comunicación, que podría manifestarse en la ejecución de planes de comunicación, se posiciona como el tema con mayor relevancia, la evolución digital queda relegada al séptimo lugar, por debajo de cuestiones como el uso del big data y los algoritmos, el acercamiento del papel de la comunicación a las decisiones directivas o la construcción y el mantenimiento de la confianza, entre otros. A lo largo del estudio, trataremos de inferir, a partir de la oferta y demanda de servicios declaradas por las agencias, en qué medida la situación en Galicia responde a los patrones establecidos por el estudio europeo para el caso español.

En tercer lugar, se ha tratado de determinar si la crisis económica iniciada en 2009, a pesar de estar cada vez más alejada en el tiempo, puede ser 
aún una circunstancia condicionante en la valoración de la comunicación y en la contratación de servicios comunicativos a las agencias, tal y como recogen otros estudios autonómicos anteriores como el de MundiNova Consultores de Comunicación (2013).

Otro aspecto cuya importancia se ha destacado en los últimos años es la comunicación con conciencia, entendida como la plena percepción y responsabilidad en la toma de decisiones de comunicación y acción por parte de las organizaciones. Este tema fue eje central del Foro Mundial de la Comunicación y las Relaciones Públicas celebrado en Madrid en 2014, organizado por Dircom y promovido por la Global Alliance for Public Relations and Communication Management. Al año siguiente, el informe sobre el estado de la comunicación en España (Dircom, 2015) incorporó este elemento dentro de su análisis. Así pues, en el presente estudio, se ha incluido este aspecto, formulando como cuarto objetivo conocer el nivel de desarrollo de la comunicación con conciencia en las organizaciones gallegas.

\section{Metodología}

\section{Desarrollo y diseño de la encuesta}

Para la realización del estudio, se empleó una técnica cuantitativa consistente en la elaboración y distribución de un cuestionario en línea, vía correo electrónico, a responsables de agencias que ofrecen servicios de comunicación y relaciones públicas en Galicia. Las preguntas fueron diseñadas en consonancia con el cuestionario elaborado para responsables de empresas e instituciones gallegas, dentro del proyecto de Dircom ya citado.

\section{Selección de la muestra y criterios de exclusión}

No existe ningún directorio específico en el que figuren las agencias que ofrecen servicios de comunicación estratégica y relaciones públicas en Galicia. Así, para tratar de definir esta oferta y actividad en la comunidad autónoma, se recurrió a cuatro repertorios en línea más genéricos en los que estas agencias podrían estar incluidas: 
- Guía de la Comunicación de la Xunta de Galicia: se trata de un directorio del Gobierno autonómico que en su apartado de "Medios de comunicación" dispone de un epígrafe dedicado a "Agencias de publicidad, comunicación y relaciones públicas", con 274 registros.

- Directorio Comunicación Galicia Digital: dispone de un apartado de "Gabinetes de prensa" en el que figuran solo cinco consultoras y de un apartado de "Agencias de publicidad" con 112 empresas.

- Briefing Galego: es un portal creado en 2009 por profesionales de la comunicación y la publicidad. En él se incluye una guía de la publicidad con 177 empresas.

- Creatividade Galega: es una organización sin ánimo de lucro que busca unir a los profesionales del sector y mejorar la calidad del trabajo creativo en Galicia. Dispone de un listado de asociados profesionales en el que se computan 48 registros.

De la suma de registros de estos cuatro directorios, se eliminaron las duplicidades y resultó un listado de 337 empresas que ofrecen todo tipo de servicios relacionados con la comunicación y la publicidad en Galicia. De este listado, se excluyeron también todas aquellas agencias o empresas que no cuentan en su oferta con servicios que tengan que ver con la comunicación estratégica y las relaciones públicas; de este modo, se prescindió de los estudios de fotografía o diseño gráfico las productoras audiovisuales, las empresas de serigrafía, las empresas de servicios en línea que se centran en el mero soporte informático y no ofrecen estrategia y las centrales de medios, con lo cual se redujo la lista a 235 empresas. Este proceso se completó con una búsqueda en Google que permitió constatar la existencia de trece agencias que operan en Galicia y que no se encontraban en ninguno de los directorios, por lo cual fueron incorporadas a la lista, que pasó a sumar 248 empresas.

En el proceso posterior de búsqueda de correos electrónicos, envío de los cuestionarios e intentos de contacto telefónico, se descartaron 78 agencias, bien por no encontrarse operativas en la actualidad (pese a figurar en uno o varios de los directorios consultados), bien por la imposibili- 
dad de establecer contacto con ellas, dado que con algunas agencias que no disponían de una dirección de correo electrónico publicada en ninguno de los directorios, ni de página web o actividad en redes sociales, no se consiguió contactar telefónicamente.

Teniendo en cuenta los datos anteriores, se envió el cuestionario a 170 agencias. Para tratar de valorar la representatividad de la muestra de agencias de las que se obtuviese respuesta, se trató de conocer la facturación de las 170 agencias seleccionadas. A través de la web wwwinfoempresa.com no se pudo acceder a información de todas ellas, pero sí se comprobó que la mayoría de las que había datos disponibles registraban una facturación inferior a EUR 2 millones y solo dos superaban esa cifra. En cuanto al número de empleados, se constató también a través del servicio de información empresarial ARDÁN (www.ardan.es/ardan/index.php) que gran parte contaba con menos de diez empleados.

\section{Administración del cuestionario}

La administración se efectuó mediante un enlace enviado por correo electrónico entre el 8 de marzo y el 24 de mayo de 2017. Al cierre del proceso, se obtuvieron 60 respuestas, de las que se descartaron dos que, aun siendo consideradas a priori, no encajaban en la muestra por su oferta de servicios. Asimismo, cabe señalar que se registró el contacto telefónico de 41 empresas que se comprometieron a responder, aunque no llegaron a hacerlo. Se constató la representatividad de las 58 agencias respecto del número de empleados (mayoritariamente inferior a 10) y de facturación (quedaron incluidas las dos agencias con facturación superior a EUR 2 millones), en relación con las 170 empresas seleccionadas.

\section{Resultados}

\section{Perfil de las agencias que ofrecen servicios de comunicación y relaciones públicas en Galicia}

La mitad de las empresas que respondieron al cuestionario se definen como agencias de servicios integrales que ofrecen, entre otros, los servicios 
tradicionalmente vinculados a la comunicación y a las relaciones públicas. Cerca de la cuarta parte (22\%) son empresas de publicidad que también se hacen cargo de este tipo de trabajos si sus clientes así se lo solicitan y el $19 \%$ se define de forma específica como agencias de comunicación y relaciones públicas. Se encuentra en la muestra un porcentaje bajo de agencias especializadas $(8,6 \%)$ que ofrecen servicios concretos como branding o comunicación digital o que trabajan ofertando servicios generales de comunicación a sectores específicos como el cultural o el de la moda.

En cuanto a su tamaño, teniendo en cuenta los criterios establecidos en el informe de la Fundación para el Análisis Estratégico y Desarrollo de la Pequeña y Mediana Enpresa (Faedpyme, 2017), el $89 \%$ pueden definirse como microempresas de nueve trabajadores o menos (incluimos los negocios unipersonales) y el $7 \%$ son pequeñas empresas, de 10 a 49 trabajadores. Solo el $4 \%$ son, en términos empresariales, medianas empresas (entre 50 y 249 trabajadores), aunque podrían calificarse de grandes agencias.

Los principales clientes del $57 \%$ de estas agencias son pymes. Solo el $10 \%$ de las agencias consideran que sus principales clientes son grandes empresas y un 12,1\% viven fundamentalmente de las administraciones públicas. El $21 \%$ no destacan ningún tipo de cliente en particular.

Por lo que respecta a la fecha de creación, un $10 \%$ de las agencias comenzaron a funcionar en 2015 o en adelante, un $26 \%$ lo hicieron entre 2010 y 2014 y un $36 \%$ entre 2000 y 2009 . Solo el $28 \%$ de las empresas fueron fundadas en el siglo XX.

\section{Perfil de los trabajadores y responsables de las agencias}

El $66 \%$ de los trabajadores de las agencias analizadas tienen entre 30 y 39 años, mientras que un $29 \%$ se encuentran en la década de los 40; un $3 \%$ tienen menos de 30 años y solo un $2 \%$ superan los 50 . En cuanto a la edad de los responsables, se incrementa el porcentaje de individuos entre 40 y 49 años (48 \%) y entre 50 y 59 años (21\%); un $29 \%$ tienen entre 30 y 39 años y solo el $2 \%$ superan los 60 (figura 1 ). 


\section{Figura 1. Distribución de trabajadores y responsables de las agencias por edad}

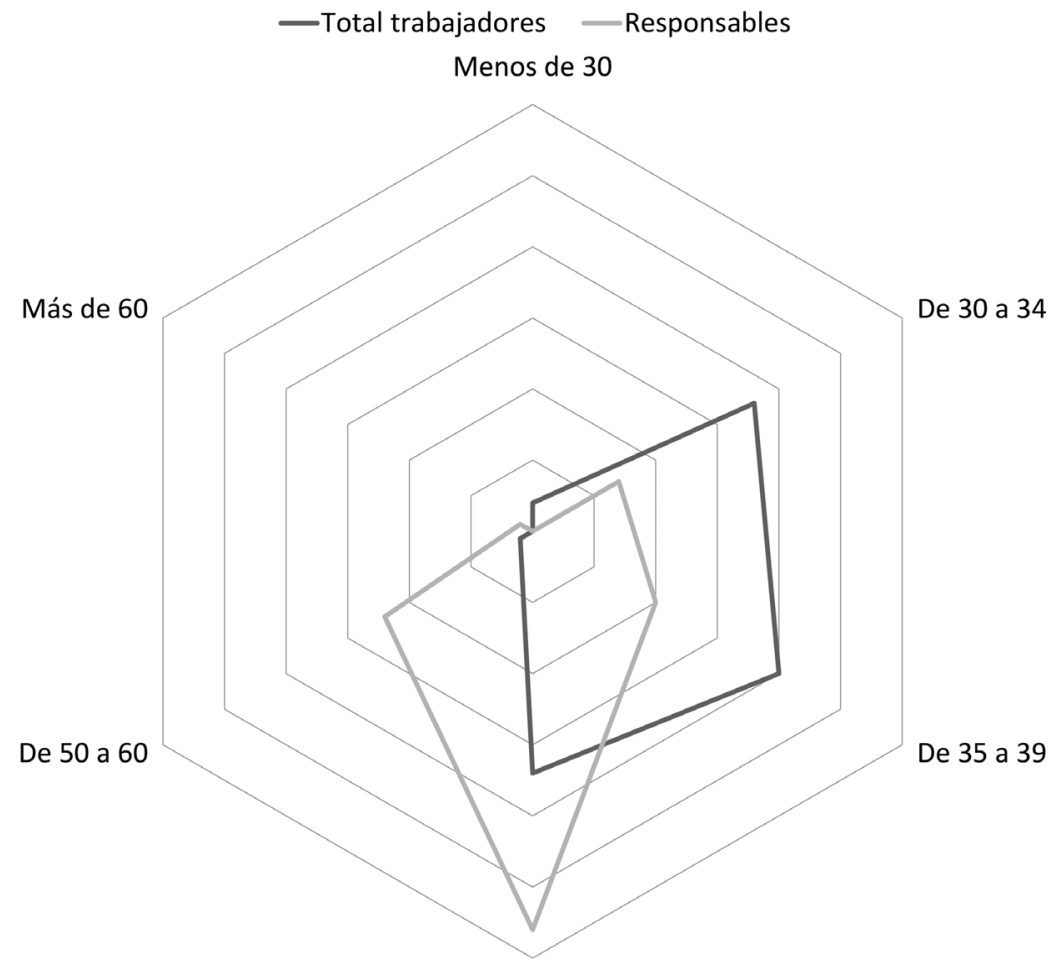

De 40 a 49

Fuente: elaboración propia.

Atendiendo al género, cabe señalar que 22 de las agencias encuestadas no han facilitado datos desglosados de número de trabajadores por género ni es posible acceder a esta información a través de la página web de la empresa. De las que sí ofrecen este dato, el 36,1 \% están integradas solo por mujeres (se trata de empresas de entre una y nueve trabajadoras), mientras que solo dos agencias $(5,6 \%)$ cuentan únicamente con hombres en su plantilla y, en ambos casos, se trata de empresas unipersonales. En términos globales, se computa un 6,8 \% de mujeres frente a un $37,2 \%$ de hombres, aunque el $60,7 \%$ de las agencias están dirigidas por hombres (figura 2). 


\section{Figura 2. Porcentaje de hombres y mujeres en las agencias}

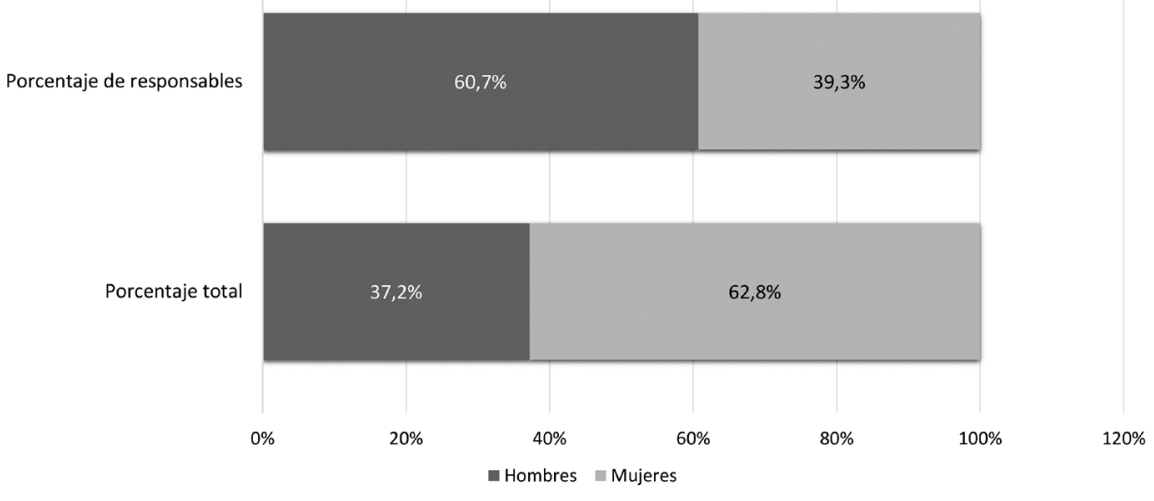

Fuente: elaboración propia.

Con respecto a la formación, cabe destacar que el 29,3 \% de los responsables de las agencias son titulados en Publicidad y Relaciones Públicas y el 15,5 \% en Periodismo. Un 10,3 \% de los responsables de las agencias consultadas no poseen formación universitaria (figura 3).

\section{Figura 3. Formación universitaria del responsable de la agencia}

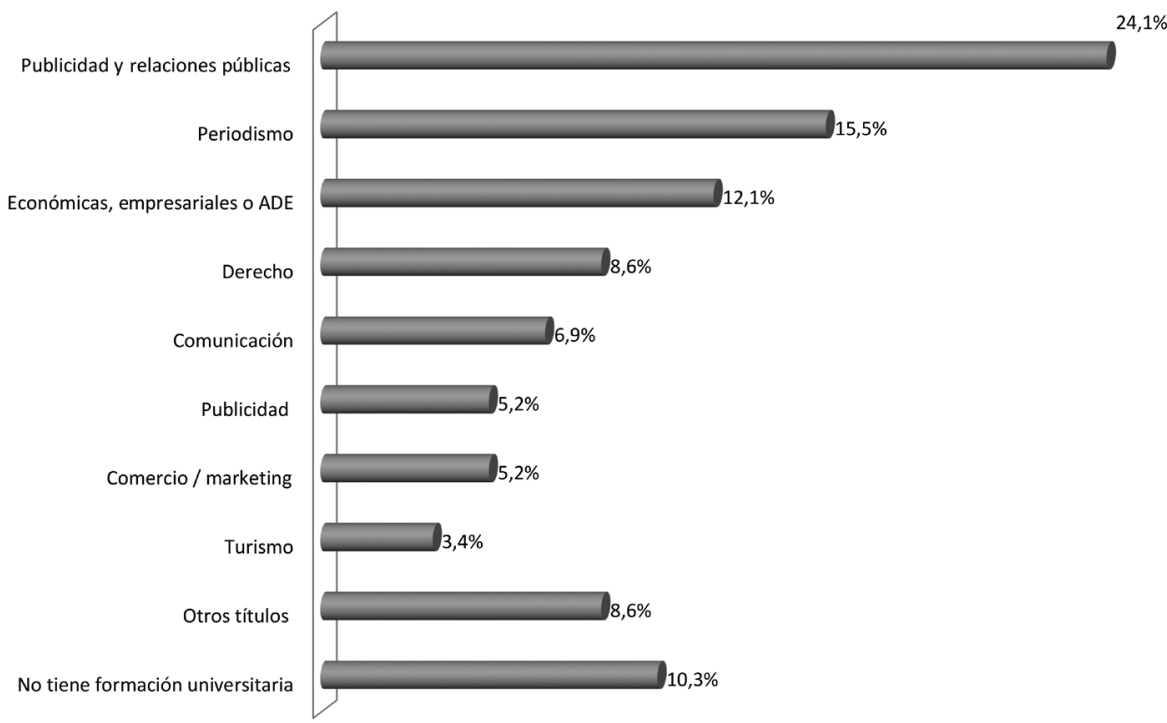

Fuente: elaboración propia. 
Asimismo, el 60,3 \% de los responsables de agencias cuenta con algún tipo de formación de posgrado. Se trata, mayoritariamente, de másteres en Comunicación o Comunicación Corporativa (13,8\%) o másteres en Economía o Empresariales (12,1 \%), aunque la diversidad de estudios de posgrado cursados es muy amplia. Solo uno de los responsables, una mujer, cuenta con un doctorado (figura 4).

\section{Figura 4. Formación de posgrado del responsable de la agencia.}

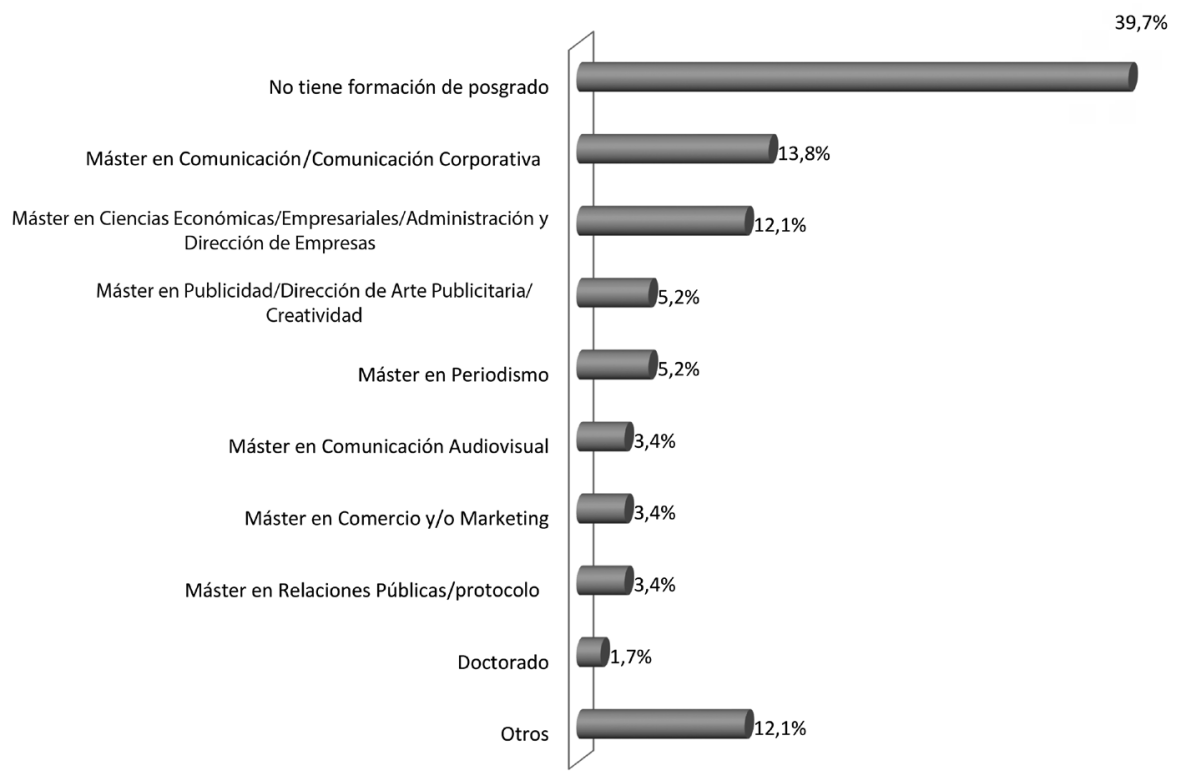

Fuente: elaboración propia.

Por último, más de la mitad de los responsables ha dedicado al menos 50 horas a la formación en el último año, aunque el 15,5\% no han dedicado ningún tiempo a esta tarea. Solo un 5,2\% de los responsables afirman haber dedicado a la formación más de 500 horas.

\section{Oferta y demanda de servicios}

Por lo que respecta a los servicios, se aprecia, en general, una alta correlación entre los servicios más ofertados y los más demandados. La mayoría de los servicios que tienen que ver con el ámbito en línea se encuentran 
tanto entre los más ofertados por las agencias como entre los más demandados por los clientes. La publicidad, ya comercial, ya de marca, y la creación de planes de comunicación son también servicios con abundante oferta y alta demanda (figura 5).

\section{Figura 5. Servicios más ofertados, más demandados y previsión de más demandados en cinco años.}

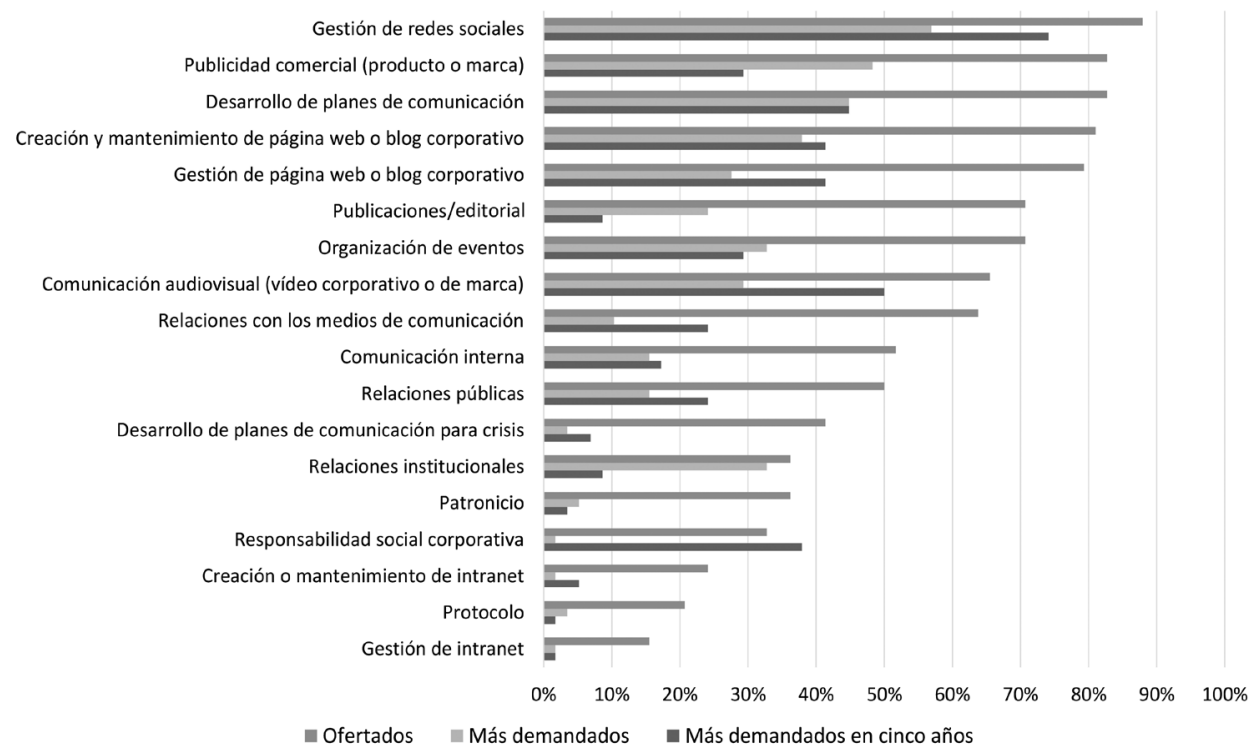

Fuente: elaboración propia.

Todas las agencias de servicios plenos ofrecen el desarrollo de planes de comunicación; también lo hacen el $62 \%$ de las agencias de publicidad, así como el $60 \%$ de las agencias especializadas; en cambio, un $27 \%$ de agencias de comunicación y relaciones públicas no lo ofrecen. En cuanto a la publicidad de producto o marca, la ofrecen entre sus servicios el $90 \%$ de las agencias de servicios plenos, todas las agencias de publicidad y las especializadas e incluso un $36 \%$ de las agencias de comunicación o relaciones públicas.

Con respecto a las expectativas para dentro de cinco años, el $74 \%$ de los encuestados coinciden en que la gestión de redes sociales será más demandada y el $50 \%$ en que lo será la comunicación audiovisual (vídeos 
corporativos o de marca). También consideran que crecerá la demanda de otros servicios como la gestión de webs y blogs corporativos, la responsabilidad social corporativa, las relaciones con los medios de comunicación o las relaciones públicas (figura 5).

\section{Tendencias}

Cuando se interroga a los responsables de las agencias acerca de la evolución de la importancia de la comunicación en los últimos cinco años, la mayoría coincide en afirmar que la comunicación es más importante ahora que antes $(71 \%)$ o algo más importante $(17 \%)$. Un $9 \%$ opinan que la relevancia se mantiene y solo un $3 \%$ consideran que la comunicación ha perdido importancia.

Con respecto a la incidencia de la crisis de 2009 en la actividad de las agencias, una amplia mayoría de los responsables identifica uno o varios aspectos en los que la crisis ha afectado a su agencia. El problema más destacado es la reducción de beneficios $(56,9 \%)$, seguido de la reducción del número de clientes $(43,1 \%)$; un porcentaje significativamente menor de encuestados apunta a la reducción de la cantidad o variedad de servicios contratados por los clientes $(27,6 \%)$ y la reducción de la plantilla $(24,1 \%)$ como consecuencias de la crisis. Algo más de la cuarta parte de los encuestados consideran, en cambio, que la crisis no ha influido en la actividad de la agencia ( $19 \%)$ o que la influencia ha sido pequeña $(8,6 \%)$, aunque cabe destacar que ninguno de ellos representa una agencia que existiese antes de 2000, cuyos responsables tienen una percepción menos optimista de los efectos de la crisis.

Cuando se pregunta qué elementos concretos de la comunicación han experimentado cambios en los últimos años, independiente de que se asocien o no al fenómeno de la crisis, la mayoría de los encuestados consideran que se han reducido los presupuestos, frente a un $19 \%$ que opinan que se han incrementado y un $12 \%$ que creen que no han variado. Por lo que respecta a los recursos humanos, también es mayoritaria la opinión de aquellos que creen que se han reducido $(55,2 \%)$ frente a los que consideran que se han incrementado $(24,1 \%)$ o que se han mantenido $(20,7 \%)$. 
En el caso de la inversión en medición, en cambio, los porcentajes se invierten (figura 6).

\section{Figura 6. Cambios en los últimos años en aspectos concretos de la actividad.}

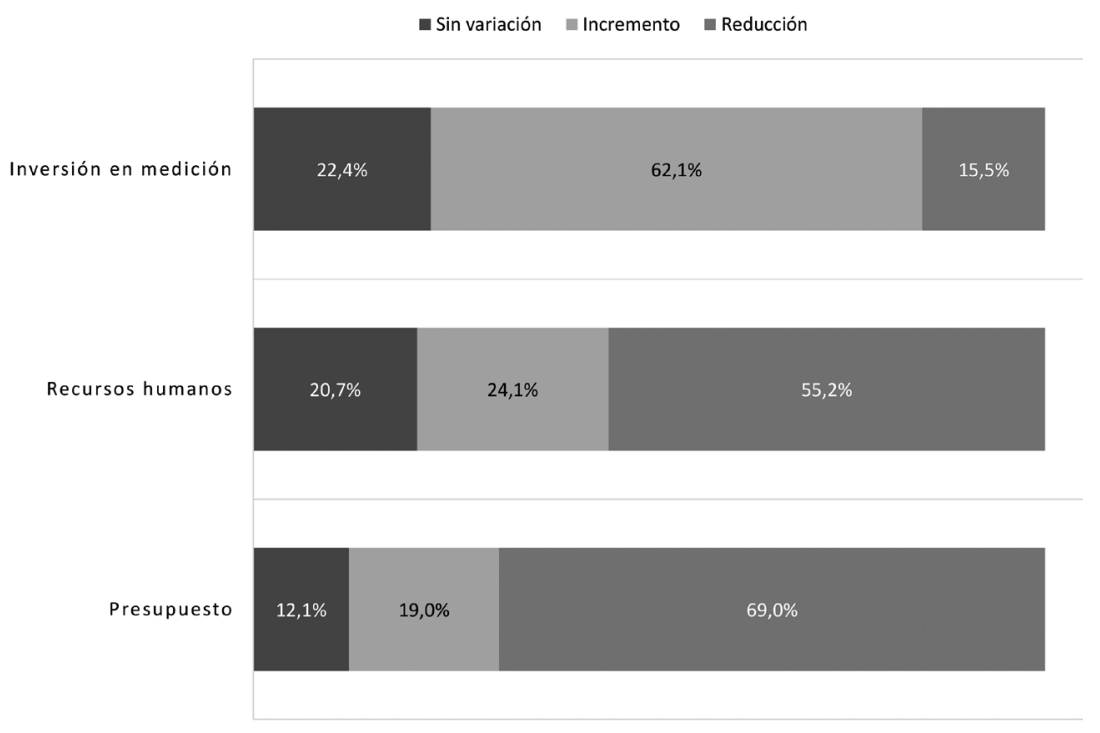

Fuente: elaboración propia.

Por último, el 65,5\% de los encuestados consideran que las organizaciones ponen en marcha la comunicación con conciencia cada vez con más frecuencia y un 3,4\% opinan que lo hacen habitualmente. Un 24,1\% indican que las organizaciones no lo hacen pero deberían, el 3,4 \% consideran que no lo hacen nunca y otro $3,4 \%$ no contestan.

\section{Discusión y conclusiones}

Mientras que estudios de ámbito nacional, como el de Dircom (2015), o relativos a otras autonomías (Rodríguez-Salcedo y Buil-Gazol, 2015), apuntan a una especialización cada vez mayor de las empresas que ofrecen servicios de comunicación, la presente investigación muestra una realidad diferente en el caso gallego. Menos de una cuarta parte de las agencias que ofrecen servicios de comunicación estratégica y relaciones públicas en Galicia son empresas orientadas específicamente a estas tareas y menos del $10 \%$ son em- 
presas especializadas, frente a una presencia elevada de agencias de servicios plenos o agencias de publicidad que, entre una oferta de servicios muy variada, incluyen los aquí descritos. Esto puede deberse a que los principales clientes de las agencias que operan en Galicia son pymes que carecen, en su mayoría, de departamento de comunicación y necesitan un servicio externo que resuelva todas las necesidades comunicativas que puedan presentar, desde la elaboración de un trabajo de diseño gráfico hasta la inserción de un anuncio en prensa, la gestión de la web o la organización de eventos. La especialización, por tanto, no es aún un valor tan destacado como puede serlo en mercados más consolidados y con agentes de mayor tamaño.

El hecho de que los clientes sean de pequeña dimensión guarda una correlación importante con el tamaño de las agencias. Son, por lo general, empresas pequeñas con estructuras flexibles (flexibilización de equipos que destacan también Estanyol y Lalueza (2014) en algunas consultoras de relaciones públicas de nivel nacional), que, cuando necesitan cubrir alguna petición de sus clientes para la cual, su plantilla no es suficiente, recurren a la subcontratación de servicios a freelances con los que colaboran habitualmente o a otras empresas. Solo dos de las agencias de la muestra pertenecen a consultoras más grandes, con sedes en varios puntos de la geografía española, recogidas en el ranking de consultoras de comunicación y relaciones públicas elaborado por PRNoticias y Torres y Carrera consultores de comunicación (2017). Estos datos revalidan los aportados por García-González y Piñeiro-Otero (2011), que indican que el 77 \% de las agencias de publicidad en Galicia tienen menos de 10 empleados y muestran que en los últimos años no se ha tendido a la creación de empresas de mayor tamaño, aunque sí hay muchas agencias de creación relativamente reciente.

En este sentido, tal y como se constató al elaborar la muestra, estamos ante un sector muy cambiante, en el que los movimientos de creación, fusión y destrucción de empresas son frecuentes y la supervivencia a lo largo de décadas es compleja. El contexto de la crisis motivó la desaparición de numerosas empresas, aunque favoreció también la aparición de otras nuevas para cubrir esos mismos nichos de mercado aparentemente vacantes. 
Solo el $28 \%$ de las empresas fueron fundadas en el siglo XX y cuentan, por tanto, con una trayectoria dilatada.

La juventud de las agencias se corresponde también con la de sus trabajadores, que tienen mayoritariamente entre 30 y 50 años, aunque, al menos en plantilla (quizá sí como becarios), no suelen integrarse personas que acaban de finalizar sus estudios. También sus responsables son jóvenes (un tercio tiene menos de 40 años) y esto puede influir positivamente en su formación.

Así, la mayoría de los responsables de las agencias consultadas poseen algún título universitario y más del $60 \%$ además un título de máster. Este dato valida la opinión del $75 \%$ de profesionales de la comunicación que consideran que la formación en este ámbito es mejor y suficiente o mucho mejor que hace veinte años (Baamonde, Puentes y Rúas, 2015, p. 158). La titulación de licenciatura o grado que más abunda es la de Publicidad y las Relaciones Públicas; este dato es representativo, porque, según los estudios de Dircom $(2010,2015)$, en los puestos de directores de comunicación de empresas e instituciones la titulación en Periodismo suele ser la que tradicionalmente alcanza mayor presencia. Esto podría indicar una mayor tendencia de los titulados en Publicidad y Relaciones Públicas a poner en marcha sus propias consultoras, ya por voluntad emprendedora, ya porque las empresas e instituciones continúan prefiriendo a titulados en Periodismo para los puestos de director de comunicación. También podría atribuirse a la existencia de una titulación en Publicidad y Relaciones Públicas en la comunidad autónoma desde 1994, aunque en Galicia también se pueden cursar estudios de Periodismo.

En cualquier caso, tal y como sí se recoge en estos estudios nacionales, la diversidad de formación de las personas que ocupan puestos de responsabilidad en este ámbito es notoria, con un porcentaje relativamente relevante de profesionales que no proceden del ámbito de la comunicación. La formación continua, asimismo, es importante para los responsables de las agencias gallegas, aunque varía considerablemente el tiempo destinado a esta tarea. 
En cuanto a la feminización de la profesión, también se produce en las agencias gallegas, aunque no alcanza los puestos de responsabilidad: más del $60 \%$ de los trabajadores de las agencias son mujeres, pero más del $60 \%$ de los responsables de esas agencias son hombres. En un contexto en el que el porcentaje de mujeres que estudian Publicidad y Relaciones Públicas o Periodismo es muy superior al de hombres (García-González y Piñeiro-Otero, 2011), el fenómeno del techo de cristal aún parece patente.

En cuanto a la oferta de servicios, cabe apuntar que en 2010 solo la mitad de las consultoras nacionales de relaciones públicas ofrecían servicios de comunicación en línea (Lalueza, 2010). Asimismo, el estudio realizado por MundiNova (2013) hace cinco años destacaba la escasa actividad en línea de las empresas gallegas e indicaba que solo un $29 \%$ de estas contrataba servicios de comunicación en línea, aunque ponía de manifiesto que "el volumen de empresas que solicitan asesoramiento en comunicación online está creciendo a un ritmo trepidante". Los datos del presente estudio confirman claramente esta previsión y la evolución positiva, ya que, en la actualidad, según las agencias, los servicios que tienen que ver con el ámbito en línea se encuentran entre los más ofertados y los más demandados y, en el caso concreto de la gestión de redes, la mayoría de las agencias augura un incremento de la demanda en los próximos cinco años.

Este no es el único aspecto en el que se manifiesta la evolución en la demanda de servicios externos. Así, si bien en 2013 los servicios de gabinete de prensa y relaciones con los medios eran los más subcontratados por las empresas, en la actualidad poco más del $10 \%$ de las agencias los consideran entre los más demandados. El estudio de 2013 indicaba, asimismo, una escasa contratación de planes integrales de comunicación y relaciones públicas, mientras que el $40 \%$ de las agencias confirman hoy que se trata de un servicio altamente solicitado.

El incremento de la relevancia de la comunicación en los últimos cinco años es un aspecto que genera un importante consenso entre los responsables de las agencias gallegas, con unos datos porcentuales muy similares a los aportados por los profesionales de la comunicación en España, según el 
estudio de Dircom (2015). Esto apunta, por tanto, a una tendencia generalizada, no limitada geográficamente ni por el tamaño empresarial.

Sin embargo, si bien, según Dircom (2015), "las consultoras y empresas de servicios de comunicación confirman el aumento de demanda de su trabajo por parte de las empresas en los últimos años y vislumbran un futuro en positivo", muchos responsables de las agencias gallegas apuntan a una reducción de los beneficios como consecuencia de la crisis y señalan también una reducción en el número de clientes.

Por otra parte, el recorte de los presupuestos que las empresas dedican a la contratación de servicios de comunicación en Galicia, identificado por el $69 \%$ de los encuestados, y destacado también en estudios anteriores a nivel gallego (MundiNova, 2013), se identifica también en el ámbito nacional (Dircom, 2015), aunque la facturación de las grandes consultoras se ha incrementado (PRNoticias y Torres y Carrera consultores de comunicación, 2017). En este contexto de recortes presupuestarios y escasez de recursos humanos que afecta, sobre todo, a pequeñas y medianas agencias, resulta especialmente relevante, en cambio, comprobar la eficacia de las acciones que se llevan a cabo. Tanto los profesionales a nivel nacional (57,14 \%, según Dircom, 2015) como los responsables de las agencias gallegas $(62,1 \%)$ apuntan un incremento en la inversión en medición que refleja un interés y esfuerzo creciente por conseguir demostrar de forma objetiva la eficacia de las acciones comunicativas.

Los resultados generales sobre la percepción de la influencia de la crisis son consistentes con los que aportan Baamonde, Puentes y Rúas (2015) en su consulta a profesionales de la comunicación gallegos, que identifican mayoritariamente como graves ( $47 \%$ ) o muy graves $(11,7 \%)$ los efectos de la crisis, mientras que solo el $11,7 \%$ declaran no haber padecido sus consecuencias.

La comunicación con conciencia se revela como una práctica cada vez más extendida, a criterio del $65 \%$ de los encuestados, aunque estos resultados son menos optimistas que los que reflejan los profesionales de las grandes empresas a nivel nacional, que consideran, en un 80,96 \% de los 
casos, que sus organizaciones aplican la comunicación con conciencia. Esta diferencia de opinión puede deberse tanto al factor geográfico como al hecho de que las organizaciones tengan una mejor percepción de sus actividades de la que tienen aquellas agencias que les prestan servicio.

Se detectan, en síntesis, algunas diferencias con respecto al ámbito nacional en cuanto a las estructuras de las agencias que ofrecen servicios de relaciones públicas y comunicación estratégica, con un alto grado de atomización y falta de especialización. En cambio, muchas de las tendencias percibidas en el ámbito nacional e internacional como la evolución hacia una mayor demanda de servicios de comunicación estratégica y en línea son extrapolables a este contexto periférico y de menor tamaño, haciendo plausible la idea de que el nivel de globalización de la actividad comunicativa es elevado $y$, si bien puede haber divergencias en aspectos puntuales, las tendencias generales son similares. La corroboración de esta hipótesis deberá efectuarse mediante estudios posteriores que analicen las estructuras, las características y la actividad de las agencias en otros contextos periféricos.

\section{Referencias}

Aldoory, L. y Toth, E. (2002). Gender discrepancies in a gendered profession: A developing theory for public relations. Journal of Public Relations Research, 14(2), 103-126. DOI: 10.1207/S1532754XJPRR1402_2

Aldoory, L. y Toth, E. (2004). Leadership and gender in public relations: Perceived effectiveness of transformational and transactional leadership styles. Journal of Public Relations Research, 16(2), 157-183. DOI: $10.1207 /$ s1532754xjprr1602_2

Asociación de Directivos de Comunicación. (2010). El estado de la comunicación en España. En Anuario de la comunicación 2010. Madrid, España: Asociación de Directivos de Comunicación. 
Asociación de Directivos de Comunicación. (2015). El estado de la comunicación en España. En Anuario de la comunicación 2015. Madrid, España: Asociación de Directivos de Comunicación.

Baamonde, X., Puentes, I. y Rúas, J. (2015). As relacións públicas en Galicia: estado da comunicación empresarial e institucional. En X. López y R. Aneiros (Coord.), A comunicación en Galicia 2015 (pp. 153-166). Santiago de Compostela, España: Consello da Cultura Galega.

Barquero Cabrero, J. D. y Castillo Esparcia, A. (2011). Marco teórico y práctico de las relaciones públicas. Barcelona, España: Furwagen.

Dauhgerty, E. L. (2014). Ethical dilemmas of female agency owners: Practices in public relations and marketing communications. Quarterly Review of Business Disciplines, 1(2), 173-190. Recuperado de http://iabdnet.org/QRBD/Volume\%201/Issue\%202/ QRBD\%20August\%2014\%20issue.pdf

Estanyol, E. y Lalueza, F. (2014). ¿Tamaño o flexibilidad? Estructura organizativa de las consultoras de relaciones públicas en España. Sphera Publica, número especial, 135-162. Recuperado de http://sphera. ucam.edu/index.php/sphera-01/article/view/205

Fundación para el Análisis Estratégico y Desarrollo de la Pequeña y Media Empresa. (2017). Análisis estratégico para el desarrollo de la pyme en España: internacionalización y orientación emprendedora. Murcia, España: Autor.

García-González, A. y Piñeiro-Otero, T. (2011). Las mujeres en el ámbito de la producción publicitaria: estudio del sector publicitario gallego desde una perspectiva de género. Revista Latina de Comunicación Social, 66, 1-21.DOI: 10.4185/RLCS-66-2011-943-505-525

Lalueza, F. (2010). ¿Más de lo mismo? Los servicios ofrecidos en España por las agencias de relaciones públicas en la era de la web 2.0. Ponencia 
presentada en $\mathrm{V}$ Congreso Internacional de Investigadores en Relaciones Públicas, Barcelona, España.

MundiNova Consultores de Comunicación. (2013). La comunicación empresarial en Galicia: gestión corporativa y entorno online. Vigo, España: MundiNova Consultores de Comunicación.

PRNoticias y Torres y Carrera consultores de comunicación (2017). PR Informe 2017. España-Portugal. Madrid, España: Autor.

Rodríguez-Salcedo, N. y Buil-Gazol, P. (2015). Las agencias de comunicación corporativa en Cataluña. En K. Matilla (ed.), Historia de la comunicación corporativa en Cataluña (pp. 205-266). Barcelona, España: UOC.

Rojas Orduña, O. (2008). Relaciones públicas: la eficacia de la influencia (2. a ed.). Madrid, España: ESIC.

Sha, B.-L. y Toth, E. L. (2005). Future professionals' perceptions of work, life, and gender issues in public relations. Public Relations Review, 31(1), 93-99. DOI: 10.1016/j.pubrev.2004.09.004

Wilcox, D. L., Cameron, G. T. y Xifra, J. (2012). Relaciones públicas: estrategias y tácticas (12. ed.). Madrid, España: Pearson.

Xifra, J. (2014). Manual de relaciones públicas e institucionales. Madrid, España: Tecnos.

Zerfass, A., Moreno, Á., Tench, R., Verčič, D. y Verhoeven, P. (2017). European Communication Monitor 2017. How strategic communication deals with the challenges of visualisation, social bots and hypermodernity: Results of a survey in 50 Countries. Brussels: EACD/EUPRERA, Quadriga Media Berlin. 